\title{
Acinetobacter meningitis: acquired infection in a neonatal intensive care unit
}

\author{
M E I MORGAN AND C A HART
}

Department of Child Health and Department of Medical Microbiology, Liverpool Maternity Hospital

SUMMARY A cluster of 4 cases of meningitis due to Acinetobacter calcoaceticus var anitratus occurred during a 5-day period in a neonatal intensive care unit. Three of the infants were preterm and all had a history of other medical problems. Initiation of intravenous therapy with carbenicillin was accompanied by clinical recovery and a bacteriological cure. Intensive bacteriological investigation failed to show a common source for the infections.

Bacterial infections are a common cause of morbidity and mortality in neonates. ${ }^{1}$ Such infections occurring after age 48 hours are increasingly due to organisms derived from the ward. ${ }^{2}$ We describe a cluster of cases of meningitis due to a rare Gramnegative cocco-bacillus which occurred in a neonatal intensive care unit.

\section{Methods}

Cerebrospinal fluid was obtained from the infants by lumbar puncture using full aseptic precautions. The fluid was examined microscopically and an aliquot was cultured aerobically $\left(10 \% \mathrm{CO}_{2}\right)$ and anaerobically. Bacteria were tentatively identified as Acinetobacter sp. by their appearance as Gramnegative cocci and the fact that they were oxidase negative. Their identity was confirmed using the API 20E system (API Ltd). Rectal, pharyngeal, umbilical, and skin swabs were obtained from infected infants and other neonates in the unit. Members of staff were screened using hand washings, throat, and rectal swabs. The inanimate moist environment was sampled by means of sterile absorbent swabs and a centrifugal air sampling device (Biotest Gmbh).

\section{Patients}

Case 1. A girl weighing $2.8 \mathrm{~kg}$ was admitted on 27 May 1981 following asphyxia at birth. After exchange transfusion for polycythaemia, generalised hypertonia with fisting developed with abdominal distension and passage of blood per rectum. A diagnosis of necrotising enterocolitis was confirmed radiologically. Intravenous therapy with gentamicin, penicillin, and metronidazole was started. During the ensuing 48 hours several convulsions with continuing cerebral irritability were noted. On microscopical examination cerebrospinal fluid (CSF) taken at the beginning of the period (28 May) appeared normal but a moderate growth of Acinetobacter calcoaceticus var anitratus was obtained on culture. On 30 May intravenous treatment with carbenicillin ( $800 \mathrm{mg} / \mathrm{kg}$ a day) was begun. A further sample of CSF obtained on 1 June was again normal on microscopical examination but Acinetobacter sp. was obtained on culture. CSF taken the next day and 7 days later was sterile on both occasions. After 4 days' treatment with carbenicillin the infant's clinical condition improved and treatment was stopped after a 3-week course. The infant's mother had no vaginal discharge but Acinetobacter sp. carriage was not specifically excluded.

Case 2. A girl of 30 weeks' gestation weighing $1.1 \mathrm{~kg}$ was admitted on 26 May 1981 after a traumatic forceps delivery. She developed occasional apnoeic attacks which were managed with aminophylline suppositories. Three days after birth she underwent an exchange transfusion for hyperbilirubinaemia, and treatment with gentamicin and penicillin was initiated because of continuing apnoeic episodes. CSF at this time was sterile. Two days later the apnoeic attacks increased in frequency and she was artificially ventilated after a prolonged episode of apnoea. CSF obtained on 31 May was blood stained but no organisms were seen. Culture of this fluid showed a moderate growth of Acinetobacter anitratus. Intravenous therapy with carbenicillin ( $800 \mathrm{mg} / \mathrm{kg}$ a day) was begun and CSF taken 2 days later was sterile. Her clinical condition improved and treatment was stopped after 3 weeks.

Case 3. A 36 weeks' gestation boy weighing $2.6 \mathrm{~kg}$ was admitted on 17 May 1981 following cyanosis after delivery. Respiratory distress syndrome was diagnosed and treated with mechanical ventilation. The course was complicated by the development of 
Table Cases of meningitis due to Acinetobacter calcoaceticus in infants

\begin{tabular}{|c|c|c|c|c|c|c|}
\hline Year & Age & $\operatorname{Sex}$ & Appearance of CSF & Blood culture & Treatment & Outcome \\
\hline $\begin{array}{l}19574 \\
19574 \\
19655 \\
19655 \\
19706\end{array}$ & $\begin{array}{l}4 \text { days } \\
6 \text { days } \\
1 \text { year } \\
1 \text { month } \\
5 \text { weeks }\end{array}$ & $\begin{array}{l}\mathbf{F} \\
\mathbf{M} \\
\mathbf{F} \\
\mathbf{M} \\
\mathbf{F}\end{array}$ & $\begin{array}{l}\text { Cloudy } \\
\text { Cloudy } \\
\text { NR } \\
\text { NR } \\
\text { Gram-positive cocci } \\
\text { Gram-negative cocci }\end{array}$ & $\begin{array}{l}\text { NR } \\
\text { NR } \\
\text { Negative } \\
\text { Negative } \\
\text { NR }\end{array}$ & $\begin{array}{l}\text { NR } \\
\text { NR } \\
\text { NR } \\
\text { NR } \\
\text { Kanamycin and } \\
\text { penicillin }\end{array}$ & $\begin{array}{l}\text { Recovered: deaf } \\
\text { Died } \\
\text { Recovered } \\
\text { Recovered: hydrocephalus } \\
\text { Recovered }\end{array}$ \\
\hline $1975^{7}$ & 2 months & $\mathbf{M}$ & $\begin{array}{l}7 \text { WBC } \\
\text { Gram-negative cocci }\end{array}$ & Negative & Gentamicin & Recovered \\
\hline 19798 & 2 weeks & $\mathbf{F}$ & $\begin{array}{l}2600 \text { WBC } \\
\text { No organisms }\end{array}$ & NR & $\begin{array}{l}\text { Gentamicin and } \\
\text { ampicillin }\end{array}$ & Recovered \\
\hline
\end{tabular}

$\mathrm{NR}=$ not recorded

extensive intraventricular haemorrhage diagnosed by ultrasound examination. CSF taken at this time (20 May) was sterile. On 31 May his colour was poor and his temperature unstable. CSF was still sterile. On 1 June he began to vomit and was pale and irritable. Blood-stained CSF was obtained but no organisms were discernible. Culture of this fluid showed a moderate growth of $A$. anitratus. Treatment with carbenicillin was begun $(800 \mathrm{mg} / \mathrm{kg}$ a day) and clinical and bacteriological improvement was rapid. This treatment was stopped after 3 weeks.

Case 4. A 30-week gestation boy weighing $0.8 \mathrm{~kg}$ was admitted on 28 April 1981 with respiratory distress syndrome. Treatment by mechanical ventilation was complicated by pneumothorax and the development of subglottal stenosis. He required prolonged artificial ventilation. He received penicillin and gentamicin until 16 May after an episode of neutropenia. On 1 June his condition deteriorated clinically with the onset of convulsions. Treatment with penicillin and gentamicin was re-started. CSF taken at this time was blood stained and no organisms were seen; however on culture a moderate growth of $A$. anitratus was obtained. Antibiotic therapy was changed to carbenicillin $(800 \mathrm{mg} / \mathrm{kg}$ a day) and continued for 3 weeks. No further convulsions occurred and subsequent culture of CSF proved sterile.

\section{Further investigations}

Each isolate had an identical 'API-pattern' and the same sensitivity pattern being sensitive to gentamicin, carbenicillin, chloramphenicol, and sulphonamides but resistant to cefuroxime and ampicillin. Acinetobacter sp. were not recovered from the blood, rectum, pharynx, or skin swabs of the infected infants. Samples of CSF from 2 infants nursed in proximity to the infected neonates were sterile and screening swabs from all the infants in the unit were negative. Rectal swabs and hand washings from 26 members of the attendant staff did not reveal acinetobacter except for hand washings from 2 nurses working in the milk kitchen. Neither of these isolates had the same antibiotic sensitivity pattern nor had they the same 'API-pattern' as the strains infecting the infants. Environmental swabs and culture of intravenous solutions were uniformly negative. At follow-up 3 months later each infant showed full recovery and there was no evidence of a gross neurological deficit.

\section{Discussion}

Acinetobacter (previously Achromobacter sp. or Herellea vaginicola) is a commensal and a rare opportunistic pathogen. Although clusters of urinary tract infections due to this organism have been described from an adult intensive care unit, ${ }^{3}$ cases of meningitis in neonates are sporadic and are rarely reported (Table). We consider that the cases reported here represented meningeal infection because the isolation of the organisms coincided with the onset of clinical signs; and initiation of appropriate therapy which is known to enter the CSF was associated with clinical and bacteriological cures. The source of these infections remains unknown, since extensive surveillance of the ward environment, both animate and inanimate, was uniformly negative. Nevertheless, we suggest that Acinetobacter calcoaceticus var anitratus be added to the list of Gram-negative opportunists which can cause outbreaks of nosocomial infection in neonatal units.

We thank Dr R W I Cooke for permission to report on patients in his care.

\section{References}

1 Hemming V G, Overall J C, Jr, Britt M R. Nosocomial infections in a newborn intensive care unit. $N$ Engl $J$ Med 1976; 294 : 1310-6. 
2 Davies $\mathbf{P}$ A. Infections with gram negative rods. J Antimicrob Chemother 1979; 5: Supplement A, 13-20.

3 French G L, Casewell M W, Roncoroni A J, Knight S, Phillips I. A hospital outbreak of antibiotic-resistant Acinetobacter anitratus: epidemiology and control. J Hosp Inf 1980; 1: 125-32.

4 Watson D G. Purulent neonatal meningitis; a study of 45 cases. J Pediatr 1957; 50: 352-60.

5 Lothe F, Griffin E. Bacterium anitratum and Mima polymorpha infection in Uganda. J Clin Pathol 1965; 18: 301-6.

- Gromisch D S, Gordon S G, Bedrosian L, Sall T. Simul- taneous mixed bacterial meningitis in an infant. Am J Dis Child 1970; 119: 284-6.

7 Odugbemi T, Ajasin M A. Pyogenic meningitis due to Bacterium anitratum. East Afr Med J 1975; 52: 219-22.

8 Yogev R. Ventriculitis from Acinetobacter calcoaceticus variant anitratus. J Neurol Neurosurg Psychiatry 1979; 42: 475-7.

Correspondence to Dr M E I Morgan, Liverpool Maternity Hospital, Oxford Street, Liverpool L7 7BN.

Received 26 February 1982

\section{Prevalence of infant colic}

\section{DAVID W HIDE AND BARRY M GUYER}

St Mary's Hospital, Newport, Isle of Wight, and Mead Johnson Nutritionals, Langley, Slough, Berks

SUMMARY Colic affected $16 \%$ of 843 infants. It was not related to breast or cows' milk feeding. Increased prevalence was noted if cereals or solids had been introduced during the first 3 months of life and in infants from professional or skilled social groupings.

Colic is a common problem in infancy. Although it is not generally associated with serious disease it may be profoundly disturbing to the child and his family. The mother's distress is exacerbated by lack of an adequate explanation. Her inability to help her infant is made worse by the implication that the colic may result from some failure in the relationship between mother and baby. Certainly a young infant with severe colic tests the affection of any member of the family exposed to it. Many hypotheses have been made about the cause of colic, sometimes called 3-month or evening colic, but few have been substantiated. Cows' milk allergy has been reported as a cause of infantile colic ${ }^{1}$ although others have suggested that colic is more common in breast-fed infants. ${ }^{2}{ }^{3}$ This study reports the prevalence of colic in infants and relates it to mode of feeding.

\section{Patients and method}

The Isle of Wight infant feeding survey was instituted in 1977 to study the pattern of feeding in an entire population born during a 12-month period. The structure of the study has been reported. ${ }^{4}$ As part of the survey, data on colic were recorded by health visitors $6,12,26$, and 52 weeks after birth. Further information was obtained from the mothers 2 years after the infant's birth. Data have been collected on 843 infants.

\section{Results}

Colic affected $135(16 \%)$ of the infants and was present in most (120) by age 6 weeks. Only 11 of the affected infants developed colic for the first time between ages 6 weeks and 3 months, the remaining 4 showing initial symptoms after this age.

Almost half $(47 \%)$ of the infants who first had colic before age 6 weeks had lost their symptoms by the time they were 3 months old. A further $41 \%$ remitted before age 6 months, leaving only $12 \%$ with symptoms persisting into the second half year of life (Figure).

Attempts to demonstrate relationships between colic and the time of day of occurrence of symptoms

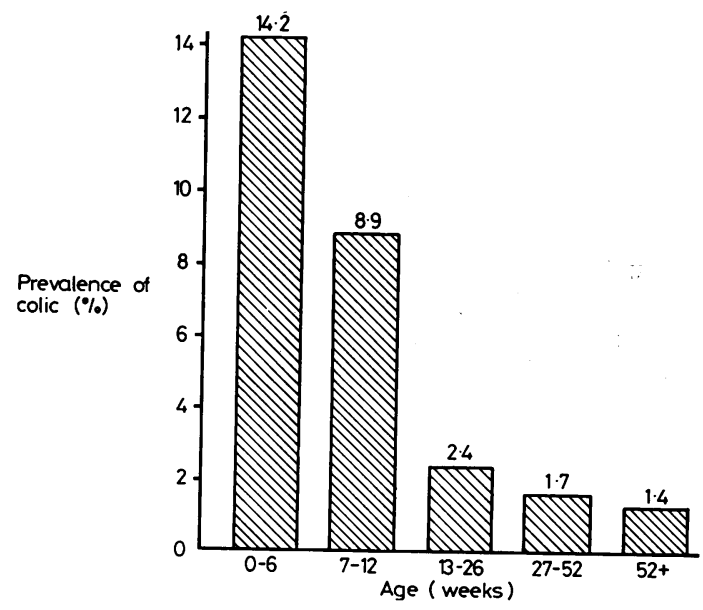

Figure Prevalence of cholic in infancy. 